\title{
A Self-Organized Energy Efficient Topology Control Protocol based on Cellular Learning Automata in Wireless Sensor Networks (SEETCLA)
}

\author{
Shekufeh.Shafeie \\ Young Researchers Club, Arak Branch \\ Department of Technology and Computer Engineering \\ Islamic Azad University \\ Arak, Iran \\ sh.shafeie@gmail.com
}

\author{
M. R. Meybodi \\ Department of Computer Engineering and Information Technology \\ Amirkabir University of Technology \\ Tehran, Iran \\ mmeybodi@aut.ac.ir
}

\begin{abstract}
Among some of sensor network properties which make it different from other networks, can refer to very high number of nodes, dynamic, and probably periodic topological changes and also some constraints in physical size of nodes, energy resource and power of processing. According to these restrictions, giving solutions and self-configurable protocols that do global tasks without requiring a central controller or manager are necessary. Topology control and node scheduling that constitute a part of the maintenance phase of self-organization protocols, are providing the main goal of this phase which is increasing network lifetime and also maintaining the infrastructure support for the network. In consideration of learning Automata's abilities such as low computational load, the ability of being used in distributed environments, with no precise information, the adaptability to changes via low environmental feedbacks and etc. and also its functionality that has some correspondence with essential methods which are used in self-organization systems, such as positive and negative feedbacks, interacting of special nodes with each other and with the environment, and probabilistic methods, results in the fact that using them is proper for improving the performance of sensor networks.
\end{abstract}

So, in this paper a neighbour based topology control protocol has been proposed, in which an irregular cellular learning automaton is mapped to network, and with it nodes which are equipped with Automata, try to adapt their selected actions with required conditions for creating a connected, energy efficient network through selecting the best radio transmission range for themselves. This approach finally forms a proper topology which causes to lower network's energy consumption in its lifetime. The exclusive characteristic of this method is, the high number of transmission ranges that each node can select as transmission radius. Simulation's results show favorite functionality of the proposed protocol in comparison with some others from the above point of view.

Index Terms - Self-Organized Protocols, Cellular Learning Automata, Energy Efficiency, Topology Control, Wireless Sensor Networks.

\section{INTRODUCTION}

Wireless sensor networks are set of large number of sensor nodes that are close to each other that scattered in the environment and each of them autonomously and in cooperation with other nodes; tries to achieve a special goal. Every node in this collection can communicate with others and gives its information to other nodes in order to finally, the under control environment's status be reported to a central point that is sink node. The principal aim in wireless sensor networks is monitoring and controlling variations of climate and physical or chemical changes in an environment of a phenomenon with deterministic region $[1,2]$. Nevertheless, since nodes work autonomously and without human interference; and are also very small physically, and have some constraints in power processing, memory capacity and power resource and etc., the aim of many researches is focused on presenting approaches that with simple control methods and with low cost, moreover than responding to requirements, can stand for some constraints such as bandwidth, limited energy, environmental interferences and etc. and can correspond with general conditions based on requirements and available wills such as, transferring high density rich in content information, long lifetime, low cost and etc.

Based on the control schema, WSNs can be non-selfconfigurable or self-configurable [2]. In the former mechanism, the sensor nodes are not able to organize on their own, but rely on a central controller to offer command to and collect information from them. This schema can only be used in small-scale networks. However, in most WSNs, the sensor nodes can autonomously maintain and establish connectivity by themselves and collaboratively fulfill sensing and control tasks. This self-configurable schema fits better in large-scale systems to perform complicated monitoring tasks and information collection and dissemination. Although selfconfigurable systems are more complicated than non-selfconfigurable ones, they are more practical for deployment in the real world, especially when the network size becomes very large. Self-configurable network protocols use some especial qualitative metrics such as connectivity for correspondence with conditions. Selecting a suitable topology which is part of maintenance phase in self-configurable protocols [18] has the most influence on efficiency of network from viewpoints of energy consumption and lifetime.

Topology control in sensor networks is the art of coordinating nodes' decisions regarding their transmitting ranges, in order to generate a network with the desired properties (e.g. connectivity), while reducing node energy consumption and/or increasing network capacity [3]. 
Investigations on Learning Automata (L.A) and Wireless sensor networks characteristics have shown that Learning Automata with consideration to properties such as low computing overload, the ability of being used in distributed environments with ambiguous information, and the ability of adaptation to environmental changes, is a very suitable model for being used in sensor networks.

Specially, with consideration to energy restriction in sensor nodes and the requirement to reduce transmissions of redundant information, to prevent energy wasting; wireless sensor networks' tendency is high to use such algorithms; that can work in a distributed manner, with local information. Because of this, In this paper a neighbour based topology control protocol, based on cellular learning Automata has been proposed (SEETCLA) ${ }^{1}$ in which, a cellular learning automata has been mapped to the network and network nodes which are in the role of network cells with its help, and with passing of time will select the best transmission range, according to conditions for themselves.

In previous presented methods the number of transmission ranges that each node could have been selected was limited and few, but in this proposed method some efforts have been made to give nodes more optional selections which are close to each other, as transmission ranges.

The rest of this paper is organized as follows. Section 2 is summary introduction of self-configuration. Section3 is an introduction of topology control and related works on it. Learning automata as a basic learning strategy used in the proposed method; will be briefly discussed in section 4 . In Section5 the problem statement is defined. In section 6 the proposed method is presented. Simulation results have been shown in section 7 . Section 8 is the conclusion.

\section{SELF ORGANIZATION INTRODUCTION}

Self-organization is the ability of the nodes deployed to locally self-configure among themselves to form a global interconnected network [19]. Energy efficiency and response time are two crucial design challenges in self-configurable sensor network protocols. Self-organization in WSNs is performed based on the two following layers [20]: first is the task allocation layer, which is responsible for coordinating, memory management, synchronizing and working as middleware. Second is the communication layer, which is responsible for routing, data management and topology control.

Several critical service requirements at various layers of the protocol stack should be considered in self-organization protocol. Some of these requirements may be conflicting and, hence, a general trade-off may need to be included in the design. Also an integrated approach with some adaptive behavior is therefore warranted in the design of selforganization protocols. From these approaches we can refer to topology control protocols which play a key role in the performance of networking services such as scheduling of transmissions, routing, flooding, and broadcasting.

One of the methods for controlling network topology is asking nodes to select their neighbors based on some special qualitative criteria, such as connectivity or available energy or both of them [19]. Another method is to ask nodes to set their transmission radio ranges for controlling their neighbor based topology.

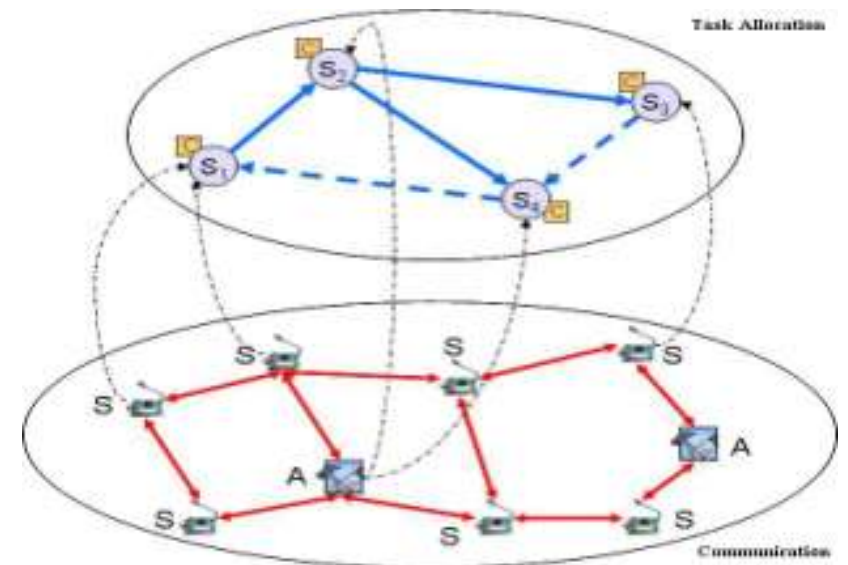

Figure1. Self-organizing supporting layers for wireless sensor network [20]

\section{TOPOLOGY CONTROL}

\section{A. Review of topology control}

The goal of topology control mechanisms is to dynamically change the nodes' transmitting range [18] and for getting into it, control the radio power level of nodes to achieve a connected and of course optimum topology; so that we can maintain some properties of the communication graph, while reducing the consumed energy by node's transceivers, and also can control the energy consumption since transceivers of nodes are one of primary sources of energy consumption in Wireless sensor networks.

These mechanisms themselves, regarding to the essence of the network and the information that each node can obtain is divided into two categories that are: homogeneous and nonhomogeneous topology control. [3,4]

In homogeneous topology control, all nodes of the network use the same transmission range $r$ and the topology control problem reduces to the problem of determining the minimum value of transmission range $r$ such that network properties like connectivity are satisfied [3,4]; but this kind of topology is not suitable enough in terms of efficiency, lifetime and strong connectivity.

In nonhomogeneous topology control, nodes are allowed to choose different transmitting ranges and independently choose their transmitting range in order to besides preserve local connectivity, have less interference too, and depending on the type of information that is used to construct the topology, it is classified into three categories [3]. First of them is locationbased approaches, that nodes know some accurate information about their positions and with the use of this information try to construct a proper topology for network.

Second is, direction-based approaches that in them it is assumed that nodes do not know their positions; but can estimate the relative direction of their neighbours. Third is, 
neighbour-based techniques that nodes have access to a minimal amount of information regarding to their neighbours, such as their I.D number.

\section{B. Related Works On neighbour based Topology control}

$\mathrm{Kneigh}^{2}$ and $\mathrm{XTC}^{3}$ protocols are two distributed topology control protocols from nonhomogeneous topology control protocols and neighbour based $[15,16]$.

The goal of Kneigh protocol is to keep at least $k$ nearer neighbours to each node. Every node increases its amount of transmission power until it can communicate with its $k$ neighbours directly.

In XTC protocol each node orders its neighbours based on the concept of link quality and distance. Then communicates with neighbours which cannot communicate with them through other nodes, and direct communication cost would be lower than indirect one.

From other topology control protocols can refer to RAA_2L that in it every node selects one from two level transmission ranges $R_{s}$ or $R_{w}$ somehow $\left(R_{w}<R_{s}\right)[6]$. If node with $R_{w}$ transmission range can communicate with $R_{s}$ transmission range neighbours (either directly or through a whisperer range neighbor); then node selects $\mathrm{R}_{\mathrm{w}}$ transmission range; otherwise it selects $R_{s}$ transmission range. In RAA_3L transmission ranges extends to three levels that every node selects one from three transmission ranges $R_{s}$ or $R_{w}$ or $R_{t}$ that it's relation are like: $\left(\mathrm{R}_{\mathrm{w}}<\mathrm{R}_{\mathrm{t}}<\mathrm{R}_{\mathrm{s}}\right)$. The used mechanism for RAA_2L is performed for two transmission ranges $R_{s}$ and $R_{t}$. If a node selects $R_{t}$ transmission range; then again the used mechanism for RAA_2L protocol will be performed for two transmission ranges $\mathrm{R}_{\mathrm{s}}$ and $\mathrm{R}_{\mathrm{t}}$.

Also, in [5] a topology control protocol based on irregular cellular learning automata (CLATC) ${ }^{4}$ has been proposed that in it an irregular cellular learning automata is mapped to network. But, each node in the network has its own individual learning automata and in cooperation with $L A$ s of its neighbor nodes, tries to choose the most suitable transmission range from three available ranges for itself; in consideration to other nodes' transmission ranges; which has better correspondence to network's conditions.

\section{LEARNING AUTOMATA}

Learning automata (L.A) is an abstract model [7,8,17] which randomly selects one action out of its finite set of actions and performs it on a random environment. Environment then evaluates the selected action and responses to the automata with a reinforcement signal. Based on selected action, and received signal, the automata updates its internal state and selects its next action. Figure 1 depicts the relationship between an automata and its environment.

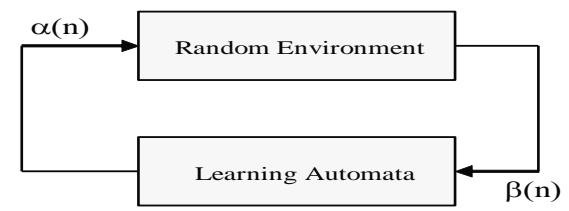

Figure2. Relationship between learning automata and its environment.

Environment can be defined by the triple $E=\{\alpha, \beta, c\}$ where $\alpha=\left\{\alpha_{1}, \alpha_{2}, \ldots, \alpha_{r}\right\}$ represents a finite input set, $\beta=\left\{\beta_{1}, \beta_{2}, \ldots, \beta_{r}\right\} \quad$ represents the output set, and $c=\left\{c_{1}, c_{2}, \ldots, c_{r}\right\}$ is a set of penalty probabilities, where each element $c_{i}$ of $c$ corresponds to one input of action $\alpha_{i}$. An environment in which $\beta$ can take only binary values 0 or 1 is referred to as $\mathrm{P}$-model environment. A further generalization of the environment allows finite output sets with more than two elements that take values in the interval $[0,1]$. Such an environment is referred to as Q-model. Finally, when the output of the environment is a continuous random variable which assumes values in the interval $[0,1]$; it is referred to as an S-model. Learning automata are classified into fixedstructure stochastic, and variable-structure stochastic. In the following, we consider only variable-structure automata.

A variable-structure automaton is defined by the quadruple $\{\alpha, \beta, p, T\}$ in which $\alpha=\left\{\alpha_{1}, \alpha_{2}, \cdots, \alpha_{r}\right\}$ represents the action set of the automata, $\beta=\left\{\beta_{1}, \beta_{2}, \cdots, \beta_{r}\right\}$ represents the input set, $p=\left\{p_{1}, p_{2}, \cdots, p_{r}\right\}$ represents the action probability set, and finally $p(n+1)=T[\alpha(n), \beta(n), p(n)]$ represents the learning algorithm. This automaton operates as follows. Based on the action probability set $p$, automaton randomly selects an action $\alpha_{i}$, and performs it on the environment. After receiving the environment's reinforcement signal, automaton updates its action probability set based on equations (1) for favorable responses, and equations (2) for unfavorable ones.

$$
\begin{aligned}
& p_{i}(n+1)=p_{i}(n)+a \cdot\left(1-p_{i}(n)\right) \\
& p_{j}(n+1)=p_{j}(n)-a \cdot p_{j}(n) \quad \forall j \quad j \neq i \\
& p_{i}(n+1)=(1-b) \cdot p_{i}(n) \\
& p_{j}(n+1)=\frac{b}{r-1}+(1-b) p_{j}(n) \quad \forall j j \neq i
\end{aligned}
$$

In these two above equations, $a$ and $b$ are reward and penalty parameters respectively. For $a=b$, learning algorithm is called $L_{R-P}{ }^{5}$. For $\mathrm{b}<<a$ it is called $L_{R \varepsilon P}{ }^{6}$. Similarly, for $b$ $=0$ the algorithm is called $L_{R-I}{ }^{7}[9]$. One types of learning automata is cellular learning automata (CLA) that in is a CA in which an LA (or multiple LA) is assigned to its every cell $[8,9]$. The LA residing in a particular cell determines its state on the basis of its action probability vector; and there is a rule that CLA operates under it. The rule of CLA and state of neighboring cells of any particular cell determine the reinforcement signal to the LA residing in that cell.

\section{PRoblem Statement}

In this paper it is assumed that every sensor node can choose a proper and deterministic value from the transmission ranges between $\left[R_{w}, R_{s}\right]$; or in other words between the low power transmission radius termed the whisperer $\operatorname{radius}\left(\mathrm{R}_{\mathrm{w}}\right)$ 
and the high power transmission radius $\left(\mathrm{R}_{\mathrm{s}}\right)$ termed the shouter radius; and there is a medium power transmission radius between $\left[R_{w}, R_{s}\right]$ which is called the speaker radius $\left(R_{t}\right)$ that its value is proportional to network density and defined with it[10]. What is being obvious from the above, is that; the $R_{w}$ transmission radius is lower than two other transmission radiuses and $R_{\mathrm{s}}$ transmission radius is higher than the two others $\left(\mathrm{R}_{\mathrm{w}}<\mathrm{R}_{\mathrm{t}}<\mathrm{R}_{\mathrm{s}}\right)$. The value of $R_{w}$ transmission radius is equal to $0.8 * \mathrm{R}_{\mathrm{t}}$, and the value of $R_{s}$ transmission radius is equal to $1.25^{*} R_{t}$. The considerable problem in this paper is selecting the most suitable transmission radius from transmission ranges between $\left[\mathrm{R}_{\mathrm{w}}, \mathrm{R}_{\mathrm{s}}\right]$ for each node; some how the required conditions for network connectivity to be conserved.

\section{THE PROPOSED METHOD}

This proposed control topology protocol that is neighbor based is performed based on cellular learning automata, and has two phases as described below: Learning and selecting the best radio range. In this model we convert the transmission range to $n$ different values; that the difference of each value with its previous value is equal to difference of each value with the next value of itself and is called Ainc or Adec and is defined as follows: Ainc $=$ Adec $=\left(\mathrm{R}_{\mathrm{s}}-\mathrm{R}_{\mathrm{w}}\right) / \mathrm{n}=\mathrm{C} * \mathrm{R}_{\mathrm{t}}$

In this relation $C$ is a constant coefficient and is equal to $\mathrm{C}=0.45 / \mathrm{n}$.

\section{A. Learning phase}

At the first of this stage, a cellular learning automata has been mapped to the network; somehow each node in the network plays role of a cell which is equipped with a L.A. and for each node according to its selected action; some neighbors would be achieved. Also, each node investigates the result of selected actions of all of its neighbors and finally, calculates the number of its neighbors which have some neighbors number out of minimum threshold $\left(\mathrm{T}_{1}\right)$ and maximum threshold $\left(\mathrm{T}_{\mathrm{h}}\right)$ values that have been achieved from [11] and is necessary to guarantee optimal connectivity. This achieved neighbors number calculating with a function called CountNotinRangeneighbors(wirelesssensor $n$ ) for each sensor node, and its return value is called Count.

Radio range of all nodes, initially is equal to each other and is $R_{t}$ which is proportional to network density. Learning automata helps the nodes to choose the most suitable radio range.

Each allocated learning automata to one cell or node has three actions: $\alpha 1, \alpha 2, \alpha 3$ for choosing, that orderly are described as follows: Increasing the radio range of each node with a constant value, Decreasing the radio range of each node with a constant value, Not to change the nodes' radio range. It is initially supposed that, the selection probability of all actions is equal to each other. In other words, according to the following relation (3), where $m$ is the number of learning automata actions: $\forall i \quad i \leq m \quad P_{i}=\frac{1}{m}$

$\alpha 1$ ( New isensorRadius $)=($ isensorRadius + Ainc $)$

$\alpha 2:($ New isensorRadius $)=($ isensorRadius - Adec $)$

$\alpha 3$ : $($ last isensorRadius $)=($ isensorRadius +0.0$)$
At first, all nodes randomly and simultaneously select one of L.A actions and then with affecting that selected actions on nodes' radio range and updating them, start broadcasting Hello messages that contain I.D number of node to all surrounding sensor nodes. Then, after selecting one of actions of automata by every cell and updating transmission ranges, return value of CountNotinRangeneighbors function or Count would be calculated and reinforcement signal for each node based on its own action and all neighbors of that node which are actually its adjacent cells would be formed.

If the Count value would be equal to 0 , reward would be given to selected action of a node; according to relation 1 because it indicates that none of its neighbors have out of range number neighbors, else selected action of a node would be given penalty according to relation 2 . Also, probability of all actions changes according to relation 1, 2 .

Reinforcement produced signal $\left(\mathrm{B}_{\mathrm{i}}\right)$, which is the reaction of environment to automata's selected action is calculated as follows:

$\mathrm{B}_{\mathrm{i}} \begin{cases}0: \text { if } \text { count }=0 & \text { : desired response } \\ 1: \text { if } \text { count }>0 & \text { :undesired response }\end{cases}$

In fact, because giving reward or penalty to one automaton's action, depends on selected action of other neighbor's automata and their updated transmission radio range, the whole mapped automata to network would be taken as cellular learning automata. Reward coefficient (a) and penalty parameter (b) is supposed to be constant and for calculating the consumed energy for transmitting and receiving packets to neighbour nodes, the proposed method in reference [12] has been used.

\section{B. Selecting the best radio range phase}

Each node will continue the learning phase and updating the radio range according to the selection that has been done (with aiding of learning automata); until one of the following conditions is happening:

- One of actions' probabilities would be higher than threshold.

- If the number of learning iterations and updating the radio range arrives to a defined threshold.

In each of these conditions, the node will choose the action which the mapped probability to it has been more than the other actions; and then will set its radio range according to that selected action.

\section{EXPERIMENTAL RESULTS}

To evaluate the performance, CLATC, RAA_2L, RAA_3L protocols [6] and homogeneous case [10] have been simulated in N.S2 simulator and the achieved results have been compared to each other and the results from the proposed 
method (SEETCLA) in the presented simulator in[13]. In simulation, nodes have been distributed in a region of $1250 * 1250$ square meters. The number of sensor nodes is ranges from 200 to 600 nodes; and also it is assumed that nodes have the same initial energy. Every node has transmission range between $\left[\mathrm{R}_{\mathrm{w}}, \mathrm{R}_{\mathrm{s}}\right]$. The medium transmission radius; $\mathrm{R}_{\mathrm{t}}$; which is proportional to network density; is assumed for 200 nodes equal to 109 meter, for 300 nodes equal to 86 meter, for 400 nodes equal to 74 meter, for 500 nodes equal to 67 meter and for 600 nodes equal to 60 meter. The $R_{w}, R_{s}$ transmissions radius are defined according to $\mathrm{R}_{\mathrm{t}}$. The energy model in these simulations is what has been expressed in [12]. Learning times for automata is limited to maximum 50 times. For this number of learning times; which is necessary for comparison with other protocols; the most suitable value of $n$ occurs in ranges between 15 to 45 , that with consideration to taking precision metric for nodes' selected transmission radius, the $n$ value in experiments has been assumed equal to 30 and 60 . However as experiments have shown too, the cost and consumed energy for learning automata's computations, against the output result that it achieves; is so low.

Considering simulation environment and network size, CLATC, RAA_2L, RAA_3L protocols [6] and the homogeneous case (HOM) and the proposed protocol (SEETCLA) have been investigated from three points of view that are: mean transmission radius, average number of neighbours for each node (the network connectivity status) and the mean residual energy, for $n$ equal to 30 and 60, and the $a$ parameter value equal to 0.3 and the initial value for parameter $b$ equal to 0.1 . The achieved results are averaged over running of the above protocols for 100 random different network configurations.

\section{A. Experiment 1}

The goal of this experiment is considering the average transmission range of network sensors for CLATC, RAA_2L, RAA_3L, HOM protocols and the proposed protocol (SEETCLA). Whatever a node's selected transmissions radius would be lower, its consumed energy would be lower too, and according to decreasing the number of neighbours, congestion happening probability will be lower too. Figure 2 shows sensor nodes' mean transmission radius for above presented protocols for different networks of different sizes. As it has shown; the proposed protocol has an approximately optimized functionality from this point into others. The HOM case has the most average transmissions radius and this is because of that all nodes in it have the same transmissions radius equal to $R_{t}$. RAA_3L protocol has less average transmissions radius than RAA_2L, because in RAA_3L every node can choose its own transmissions radius from three available transmissions ranges; while in RAA_2L protocol every node chooses its own transmissions radius from two available transmissions ranges.

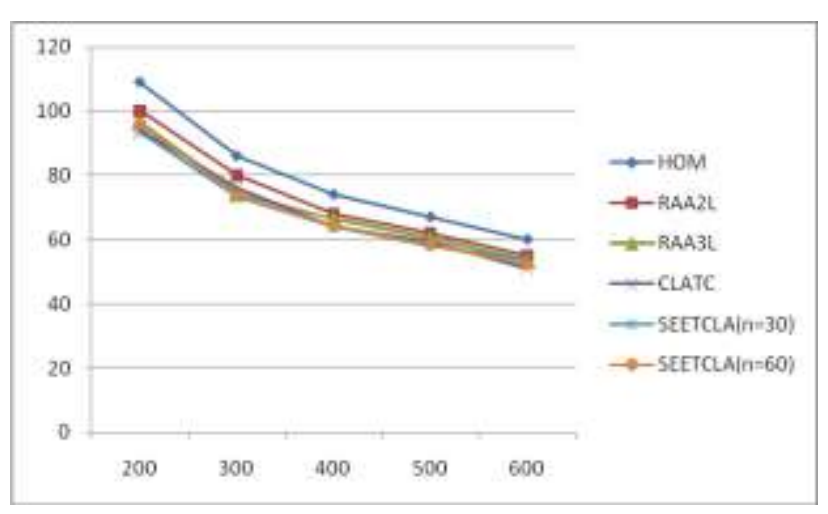

Figure 3. Average transmission radius of network's nodes for CLATC, RAA_2L, RAA_3L, HOM and proposed protocols in different network sizes.

\section{B. Experiment 2}

In this experiment the average residual energy of nodes in the network for CLATC, RAA_2L, RAA_3L protocols and the proposed protocol (SEETCLA) have been investigated. Average residual energy of network's nodes for different networks of different sizes is being observed in figure4. As it has shown the average residual energy of sensor nodes with all protocols is very high and near to initial energy of nodes, which is one joule. In spite of; the consumed energy of proposed protocol is approximately many times more than RAA_2L, RAA_3L protocols (because of existence of learning phase in the proposed protocol), but; this consumed energy in comparison with total energy of every node is so low. Residual energy increment with increasing the number of sensor nodes in network is because of transmission range decrement in networks with higher density. With consideration to indications; can deduce that evaluated protocols without consuming much more energy; give a proper topology.

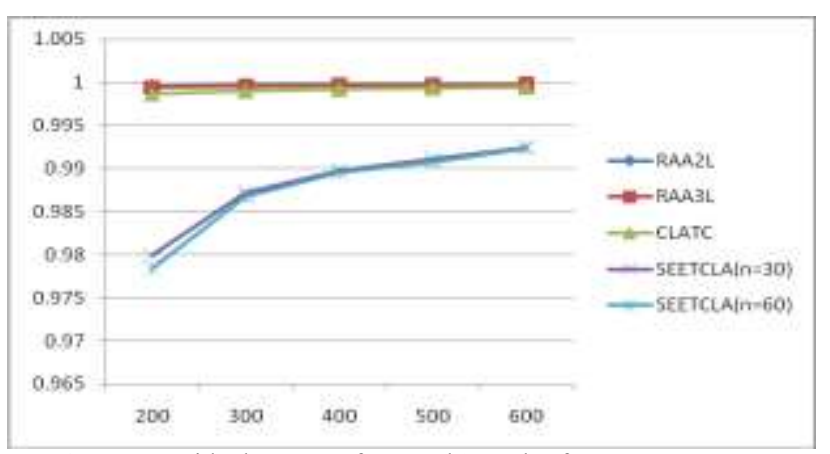

Figure 4. Average residual energy of network's nodes for CLATC, RAA_2L, RAA_3L, HOM and proposed protocols in different network sizes.

\section{Experiment 3}

The goal of this experiment is determining the number of proper sub ranges; or in other words determining better values for $n$, concerning the number of learning algorithm iterations running for network. Whatever the higher value for $n$ to be selected, the transmission range would be divided into more sub ranges and it causes more selections as radio range to be given to every node, and the precision of each node in the selection would be increased. Meanwhile $\left(\mathrm{R}_{\mathrm{s}}-\mathrm{R}_{\mathrm{w}} / \mathrm{n}\right)$ as a 
coefficient, which is the same as $C$ has direct effect on selected actions of learning automata (increment and decrement). In this experiment the proposed protocol (SEETCLA) has been investigated for different values of $n$ equal to $1,5,15,30,45,60,100,250,500,1000$ from three points of view that are: mean transmission radius, average number of neighbours for each node (the network connectivity situation) and the mean residual energy, for number of nodes in the network equal to 300 , and maximum times of learning equal to 50 times. Results have shown that when the number of learning times is equal to 50, we have the best results in a mid range between 30 and 60; however the $n$ value equal to 100 was proper too, but we have left it for the reason of consuming much more energy than other values and without achieving to considerable results.

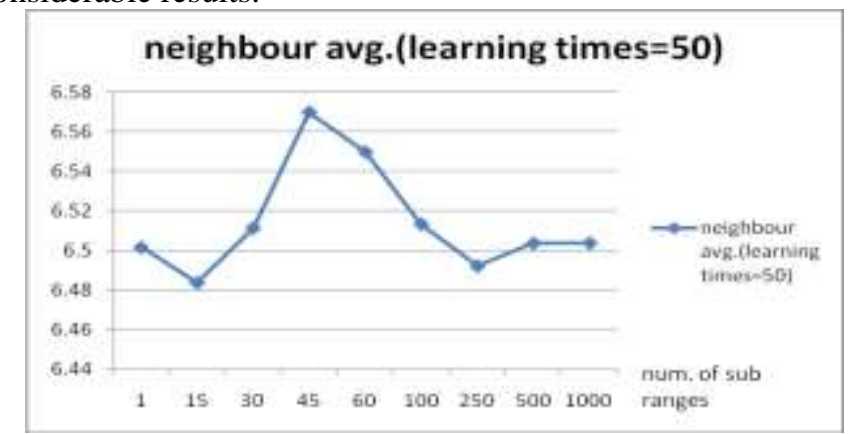

Figure 5. Average number of network nodes' neighbours for proposed protocol for 300 nodes and maximum learning times equal to 50 in different $n$ numbers.

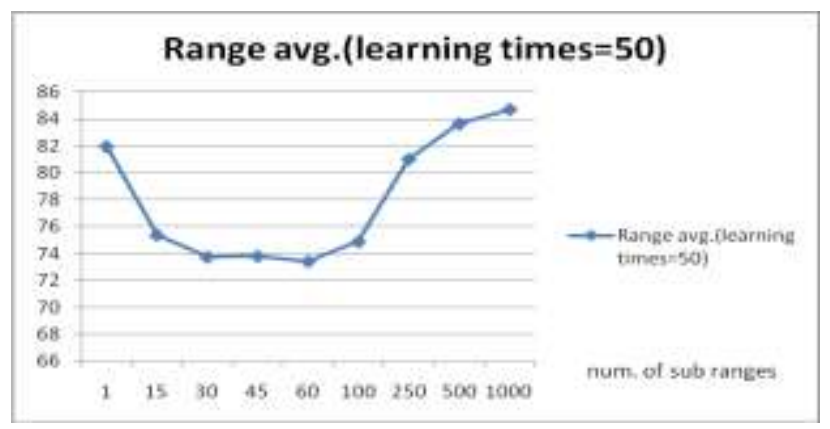

Figure 6. Average transmission radius of network nodes for proposed protocol for 300 nodes and maximum learning times equal to 50 in different $n$ numbers

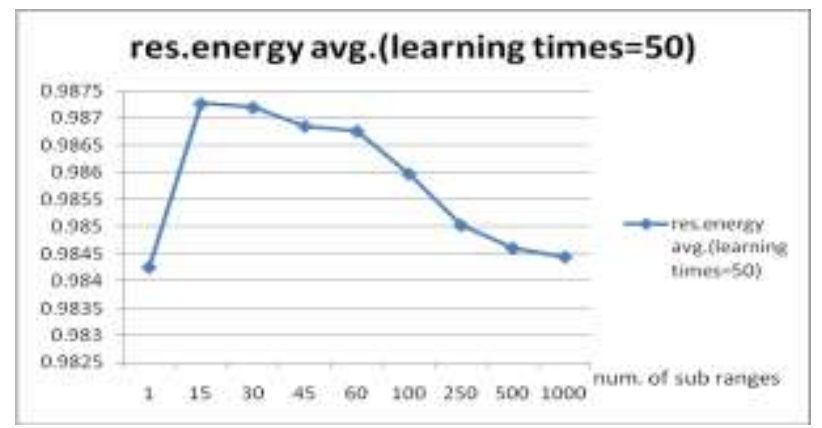

Figure7. Average residual energy of network nodes for proposed protocol for 300 nodes and maximum learning times equal to 50 in different $n$ numbers.

As a result, by increasing the number of selected sub ranges, in other words $n$, we should also increase the number of learning times too; so that we can get a desired conclusion; but this task being along with more energy consumption.

\section{CONCLUSION}

In this paper a neighbour based topology control protocol and energy efficient self-organizing based on cellular learning automata is proposed, that in it nodes which are in place of cells, in spite of that, by using learning automata and with negligible energy consumption, select proper transmission radius according to required conditions for preserving connectivity for themselves, but on the other hand, they get to an adequate topology that will have the lowest energy consumption as possible for communication between nodes in the network's life time; if a balance between the required precise values which we want to take as transmission ranges with learning times and energy consumption, would be taken into cosideration. Also, the proposed protocol, SEETCLA, with two different numbers of sub ranges has been simulated, and has been compared with other similar protocols. The results of comparisons have expressed the desired functionality of this proposed protocol into other protocols.

\section{ACKNOWLEDGMENT}

We thank M.Esnaashari at soft computing laboratory of Amirkabir University for his valuable suggestions.

\section{REFERENCES}

[1] Akyildiz I. F., Su W., Sankarasubramaniam Y. and Cayircl E., " $A$ survey on sensor networks", in: Proceedings of the IEEE Communication Magazine, Vol. 40, pp. 102-114, August 2002.

[2] Ilyas M. and Mahgoub I., "Handbook of Sensor Networks: Compact Wireless and Wired Sensing Systems", in: Proceedings of the CRC Press, London, Washington, D.C., 2005.

[3] Santi P., "Topology Control in Wireless Ad Hoc and Sensor Networks", Wiley, 2005.

[4] Santi P., "Topology Control in Wireless Ad Hoc and Sensor Networks", ACM Computer Survey, Vol. 37, No. 2, pp. 164-194, 2005.

[5] Abolhassani, S., Esnaashari, M. and Meybodi, M. R., "A Cellular Learning Automata based Topology Control Protocol for Sensor Networks", Proceedings of 14th Annual CSI Computer Conference of Iran, Amirkabir University of Technology, Tehran, Iran, March 10-11, 2009.

[6] Venuturumilli A. and Minai A. A.,"'Obtaining Robust Wireless Sensor Networks Through Self-Organization of Heterogeneous connectivity", in: Proceedings of the 2006 International Conference on Complex Systems (ICCS'06), Boston, MA, June 2006.

[7] Narendra K. S. and Thathachar M. A. L, "Learning Automata: An Introduction”, Englewood Cliffs, NJ: Prentice Hall, 1989.

[8] Beigy H. and Meybodi M. R., "A Mathematical Framework for Cellular Learning Automata", Advances on Complex Systems, Vol. 7, No. 3, pp. 1-25, 2004

[9] Thathachar M. A. L. and Sastry P. S., “Varieties of Learning Automata: An Overview", IEEE Transaction on Systems, Man, and Cybernetics-Part B: Cybernetics, Vol. 32, No. 6, PP. 711-722, 2002.

[10] Stauffer D. and Aharony A., "Introduction to Percolation Theory", London: Taylor \& Francis, 1994.

[11] L. Kleinrock and J. A. Silvester, "Optimum transmission radii in packet radio networks or why six is a magic number," in National Telecommunications Conference. Birmingham, Alabama: IEEE, Dec. 1978, pp. 4.3.1-4.3.5.

[12] Heinzelman, W., Chandrakasan, A. and Balakrishnan, H., "EnergyEfficient Communication Protocol for Wireless Microsensor Networks,"in HICSS 2000.

[13] http://www.djstein.com/projects/index.html.

[14] M. Meybodi and S. M. Abolhasani "Usage of Learning Automata for Routing, Fault Tolerance and Topology Control in Wireless Sensor Network." Vol. Master of Science Tehran Amirkabir University of Technology (Polytechnic), 2008. 
[15] Wattenhofer R. and Zollinger A., "XTC: a practical topology control algorithm for ad-hoc networks". in: Proceedings of the 18th International Parallel and Distributed Processing Symposium, pp. 2-16, 26-30 April 2004.

[16] Blough D., Leoncini M., Resta G. and Santi P., “The k-neighbors protocol for symmetric topology control in ad hoc networks", in: Proceedings of the ACM MobiHoc 03, pp. 141-152, 2003.

[17] K. Najim and A. S. Poznyak, "Learning Automata: Theory and Application", Tarrytown, NY: Elsevier Science Ltd., 1994.
[18] Mo Li, Baijian Yang "A Survey on Topology issues in Wireless Sensor Network", In Proceedings of the 2006 International Conference on Wireless Networks (ICWN), Las Vegas, Nevada, USA, June 2006.

[19] Jie Wu, Handbook on Theoretical and Algorithmic Aspects of Sensor," Ad Hoc Wireless,and Peer-to-Peer Networks", (C) 2006 by Taylor \& Francis Group, LLC.

[20] Falko Dressler," Self-Organization in Sensor and Actor Networks", John Wiley \& Sons 2007.

${ }^{1}$ Self organized Energy Efficient Topology Control Protocol based on Cellular Learning Automata (SEETCLA)

${ }^{2} k$-neighbors

${ }^{3}$ Extreme Topology Control

${ }^{4}$ Cellular Learning Automata Topology Control

${ }^{5}$ Linear Reward Penalty

${ }^{6}$ Linear Reward Epsilon Penalty

${ }^{7}$ Linear Reward Inaction 